\title{
An inverse matrix formula in the right-quantum algebra
}

\author{
Matjaž Konvalinka \\ Department of Mathematics \\ Massachusetts Institute of Technology, Cambridge, MA 02139, USA \\ konvalinka@math.mit.edu \\ http://www-math.mit.edu/ ${ }^{k}$ onvalinka/
}

Submitted: Sep 20, 2007; Accepted: Jan 23, 2008; Published: Feb 4, 2008

Mathematics Subject Classification: 15A09 (primary), 05A15 (secondary)

\begin{abstract}
The right-quantum algebra was introduced recently by Garoufalidis, Lê and Zeilberger in their quantum generalization of the MacMahon master theorem. A bijective proof of this identity due to Konvalinka and Pak, and also the recent proof of the right-quantum Sylvester's determinant identity, make heavy use of a bijection related to the first fundamental transformation on words introduced by Foata. This paper makes explicit the connection between this transformation and right-quantum linear algebra identities; we give a new, bijective proof of the right-quantum matrix inverse theorem, we show that similar techniques prove the right-quantum Jacobi ratio theorem, and we use the matrix inverse formula to find a generalization of the (right-quantum) MacMahon master theorem.
\end{abstract}

\section{Introduction}

Combinatorial linear algebra is a beautiful and underdeveloped part of enumerative combinatorics. The underlying idea is very simple: one takes a matrix identity and views it as an algebraic result over a (possibly non-commutative) ring. Once the identity is translated into the language of words, an explicit bijection or an involution is employed to prove the result. The resulting combinatorial proofs are often insightful and lead to extensions and generalizations of the original identities, often in unexpected directions.

A tremendous body of literature exists on quantum linear algebra, i.e. on quantum matrices. Without going into definitions, history and technical details let us mention Manin's works [Man88, Man89]. Recently, the work of Garoufalidis, Lê and Zeilberger [GLZ06] suggested that certain linear algebra identities (such as the celebrated MacMahon master theorem) are valid in the more general setting of $q$-right-quantum matrices (right-quantum 
matrices in their terminology). In a series or papers [FH, FH07a, FH07b], Foata and Han reproved the theorem, found interesting further extensions and an important ' $1=q$ ' principle which allows easy algebraic proofs of certain $q$-equations (implicitly based on the Gröbner bases of the underlying quadratic algebras). In a different direction, Hai and Lorenz established the quantum master theorem by using the Koszul duality [HL07], thus suggesting that MacMahon master theorem can be further extended to Koszul quadratic algebras with a large group of (quantum) symmetries. We refer to [KP07] for further references, details and the first bijective proof of the right-quantum MacMahon theorem, and some further generalizations. The approach there serves as a basis for [Kon07], in which the right-quantum Sylvester's determinant identity is proved by similar means.

The first result of this paper (Theorem 2.3) is a non-commutative algebraic identity, whose proof, presented in Section 3, is a generalization of proofs of crucial arguments in [KP07, Kon07], and which has numerous applications to right-quantum linear algebra identities. The applications presented are:

- the right-quantum matrix inverse formula (Theorem 4.1) in Section 4,

- the right-quantum Jacobi ratio theorem (Theorem 5.3) in Section 5,

- a generalization of the right-quantum MacMahon master theorem (Theorem 6.1) in Section 6.

The method gives new bijective proofs of the matrix inverse formula and Jacobi ratio theorem in the commutative case, and Theorem 6.1 appears to be new even for commutative matrices. As an example, we see that it implies the following Dixon-style identity:

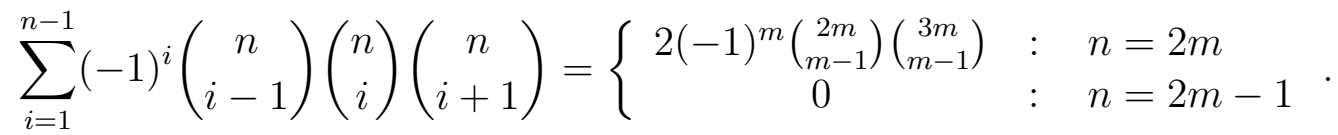

The method of proof of Theorem 2.3 is related to the first fundamental transformation described by Foata in [Foa65].

\section{Notation and the fundamental transformation}

Denote by $\mathcal{A}$ the $\mathbb{C}$-algebra of formal power series with non-commuting variables $a_{i j}$, $1 \leq i, j \leq m$. Elements of $\mathcal{A}$ are infinite linear combinations of words in variables $a_{i j}$ (with coefficients in $\mathbb{C}$ ).

Words in these variables are often written as biwords, i.e. as words in the alphabet $\left(\begin{array}{l}i \\ j\end{array}\right)$, $1 \leq i, j \leq m$, see for example [FH]; with this notation, the expression $a_{23} a_{14} a_{22} a_{41} a_{13}$

is written as $\left(\begin{array}{l}21241 \\ 34213\end{array}\right)$. In this paper, however, as in [KP07, Kon07], we represent such expressions graphically as follows.

We consider lattice steps of the form $(x, i) \rightarrow(x+1, j)$ for some $x, i, j \in \mathbb{Z}, 1 \leq i, j \leq m$. We think of $x$ being drawn along the $x$-axis, increasing from left to right, and refer 


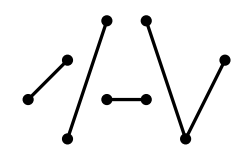

Figure 1: A graphical representation of $a_{23} a_{14} a_{22} a_{41} a_{13}$

to $i$ and $j$ as the starting height and ending height, respectively. We identify the step $(x, i) \rightarrow(x+1, j)$ with the variable $a_{i j}$. Similarly, we identify a finite sequence of steps with a word in the alphabet $\left\{a_{i j}\right\}, 1 \leq i, j \leq m$, i.e. with an element of the algebra $\mathcal{A}$. Figure 1 represents $a_{23} a_{14} a_{22} a_{41} a_{13}$.

The type of the sequence $a_{i_{1} j_{1}} a_{i_{2} j_{2}} \cdots a_{i_{n} j_{n}}$ is defined to be $(\mathbf{p} ; \mathbf{r})$ for $\mathbf{p}=\left(p_{1}, \ldots, p_{m}\right)$ and $\mathbf{r}=\left(r_{1}, \ldots, r_{m}\right)$, where $p_{k}$ (respectively $r_{k}$ ) is the number of $k$ 's among $i_{1}, \ldots, i_{n}$ (respectively $j_{1}, \ldots, j_{n}$ ). If $\mathbf{p}=\mathbf{r}$, we call the sequence balanced.

Take non-negative integer vectors $\mathbf{p}=\left(p_{1}, \ldots, p_{m}\right)$ and $\mathbf{r}=\left(r_{1}, \ldots, r_{m}\right)$ with $\sum p_{i}=$ $\sum r_{i}=n$, and a permutation $\pi \in S_{m}$. An ordered sequence of type $(\mathbf{p} ; \mathbf{r})$ with respect to $\pi$ is a sequence $a_{i_{1} j_{1}} a_{i_{2} j_{2}} \cdots a_{i_{n} j_{n}}$ of type $(\mathbf{p} ; \mathbf{r})$ such that $\pi^{-1}\left(i_{k}\right) \leq \pi^{-1}\left(i_{k+1}\right)$ for $k=$ $1, \ldots, n-1$. Clearly, there are $\left(\begin{array}{c}n \\ r_{1}, \ldots, r_{m}\end{array}\right)$ elements in $\mathbf{O}^{\pi}(\mathbf{p} ; \mathbf{r})$. Denote the set of ordered sequence of type $(\mathbf{p} ; \mathbf{r})$ with respect to $\pi$ by $\mathbf{O}^{\pi}(\mathbf{p} ; \mathbf{r})$.

A back-ordered sequence of type $(\mathbf{p} ; \mathbf{r})$ with respect to $\pi$ is a sequence $a_{i_{1} j_{1}} a_{i_{2} j_{2}} \cdots a_{i_{n} j_{n}}$ of type $(\mathbf{p} ; \mathbf{r})$ such that $\pi^{-1}\left(j_{k}\right) \geq \pi^{-1}\left(j_{k+1}\right)$ for $k=1, \ldots, n-1$. Denote the set of back-ordered sequences of type $(\mathbf{p} ; \mathbf{r})$ with respect to $\pi$ by $\overline{\mathbf{O}}^{\pi}(\mathbf{p} ; \mathbf{r})$. There are $\left(\begin{array}{c}n \\ p_{1}, \ldots, p_{m}\end{array}\right)$ elements in $\overline{\mathbf{O}}^{\pi}(\mathbf{p} ; \mathbf{r})$.

EXAmple 2.1 For $m=3, n=4, \mathbf{p}=(2,1,1), \mathbf{r}=(0,3,1)$ and $\pi=231, \mathbf{O}^{\pi}(\mathbf{p} ; \mathbf{r})$ is

$$
\left\{a_{22} a_{32} a_{12} a_{13}, a_{22} a_{32} a_{13} a_{12}, a_{22} a_{33} a_{12} a_{12}, a_{23} a_{32} a_{12} a_{12}\right\} \text {. }
$$

For $m=3, n=4, \mathbf{p}=(2,2,0), \mathbf{r}=(1,2,1)$ and $\pi=132, \overline{\mathbf{O}}^{\pi}(\mathbf{p} ; \mathbf{r})$ is

$$
\left\{a_{12} a_{12} a_{23} a_{21}, a_{12} a_{22} a_{13} a_{21}, a_{12} a_{22} a_{23} a_{11}, a_{22} a_{12} a_{13} a_{21}, a_{22} a_{12} a_{23} a_{11}, a_{22} a_{22} a_{13} a_{11}\right\} .
$$

Figure 2 shows some ordered sequences with respect to 1234 and 2314, and back-ordered sequences with respect to 1234 and 4231 .

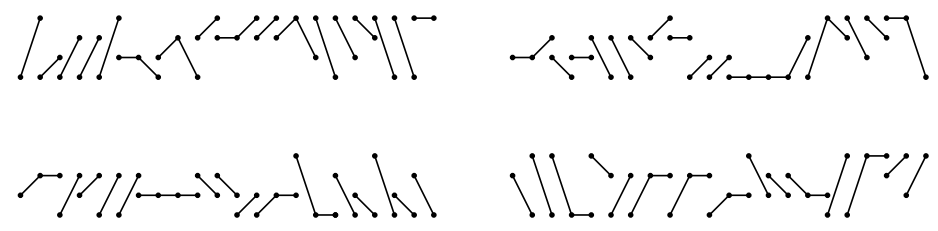

Figure 2: Some ordered and back-ordered sequences. 
We abbreviate $\mathbf{O}^{\pi}(\mathbf{p} ; \mathbf{p})$ and $\overline{\mathbf{O}}^{\pi}(\mathbf{p} ; \mathbf{p})$ to $\mathbf{O}^{\pi}(\mathbf{p})$ and $\overline{\mathbf{O}}^{\pi}(\mathbf{p})$, respectively; and if $\pi=\mathrm{id}$, we write simply $\mathbf{O}(\mathbf{p} ; \mathbf{r})$ and $\overline{\mathbf{O}}(\mathbf{p} ; \mathbf{r})$.

If each step in a sequence starts at the ending point of the previous step, we call such a sequence a lattice path. A lattice path with starting height $i$ and ending height $j$ is called a path from $i$ to $j$.

Take non-negative integer vectors $\mathbf{p}=\left(p_{1}, \ldots, p_{m}\right)$ and $\mathbf{r}=\left(r_{1}, \ldots, r_{m}\right)$ with $\sum p_{i}=$ $\sum r_{i}=n$, and a permutation $\pi \in S_{m}$. Define a path sequence of type $(\mathbf{p} ; \mathbf{r})$ with respect to $\pi$ to be a sequence $a_{i_{1} j_{1}} a_{i_{2} j_{2}} \cdots a_{i_{n} j_{n}}$ of type $(\mathbf{p} ; \mathbf{r})$ that is a concatenation of lattice paths with starting heights $i_{k_{s}}$ and ending heights $j_{l_{s}}$ so that $\pi^{-1}\left(i_{k_{s}}\right) \leq \pi^{-1}\left(i_{t}\right)$ for all $t \geq k_{s}$, and $i_{t} \neq j_{l_{s}}$ for $t>l_{s}$. Denote the set of all path sequences of type $(\mathbf{p} ; \mathbf{r})$ with respect to $\pi$ by $\mathbf{P}^{\pi}(\mathbf{p} ; \mathbf{r})$.

Similarly, define a back-path sequence of type $(\mathbf{p} ; \mathbf{r})$ with respect to $\pi$ to be a sequence $a_{i_{1} j_{1}} a_{i_{2} j_{2}} \cdots a_{i_{n} j_{n}}$ of type $(\mathbf{p} ; \mathbf{r})$ that is a concatenation of lattice paths with starting heights $i_{k_{s}}$ and ending heights $j_{l_{s}}$ so that $\pi^{-1}\left(j_{l_{s}}\right) \leq \pi^{-1}\left(j_{t}\right)$ for all $t \leq l_{s}$, and $j_{t} \neq i_{k_{s}}$ for $t<k_{s}$. Denote the set of all back-path sequences of type $(\mathbf{p} ; \mathbf{r})$ by $\overline{\mathbf{P}}^{\pi}(\mathbf{p} ; \mathbf{r})$.

EXAMPLE 2.2 Figure 3 shows some path sequences with respect to 2341 and 3421 , and back-path sequences with respect to 1324 and 4321. The second path sequence and the second back-path sequence are balanced.

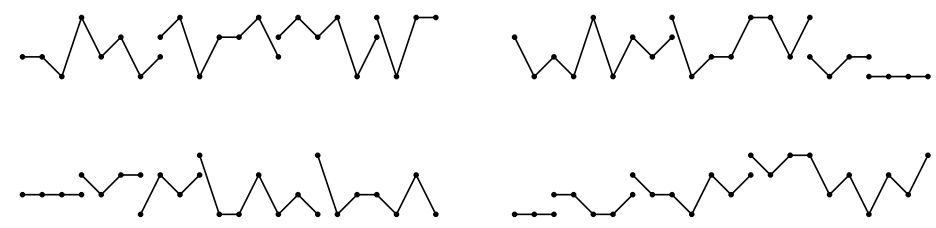

Figure 3: Some path and back-path sequences.

We abbreviate $\mathbf{P}^{\pi}(\mathbf{p} ; \mathbf{p})$ and $\overline{\mathbf{P}}^{\pi}(\mathbf{p} ; \mathbf{p})$ to $\mathbf{P}^{\pi}(\mathbf{p})$ and $\overline{\mathbf{P}}^{\pi}(\mathbf{p})$; and if $\pi=$ id, we write simply $\mathbf{P}(\mathbf{p} ; \mathbf{r})$ and $\overline{\mathbf{P}}(\mathbf{p} ; \mathbf{r})$. Note that a (back-)path sequence of type $(\mathbf{p} ; \mathbf{p})$ is a concatenation of lattice paths with the same starting and ending height.

For a word $w=i_{1} i_{2} \ldots i_{n}$, say that $(k, l)$ is an inversion of $u$ if $k<l$ and $i_{k}>i_{l}$, and write inv $u$ for the number of inversions of $u$. For $\alpha=a_{i_{1} j_{1}} a_{i_{2} j_{2}} \cdots a_{i_{n} j_{n}}$, write inv $\alpha=$ $\operatorname{inv}\left(j_{1} j_{2} \ldots j_{n}\right)-\operatorname{inv}\left(i_{1} i_{2} \ldots i_{n}\right)$. Furthermore, define

$$
\begin{aligned}
& O^{\pi}(\mathbf{p} ; \mathbf{r})=\sum_{\alpha \in \mathbf{O}^{\pi}(\mathbf{p} ; \mathbf{r})} \alpha, \quad \bar{O}^{\pi}(\mathbf{p} ; \mathbf{r})=\sum_{\alpha \in \overline{\mathbf{O}}^{\pi}(\mathbf{p} ; \mathbf{r})}(-1)^{\text {inv } \alpha} \alpha, \\
& P^{\pi}(\mathbf{p} ; \mathbf{r})=\sum_{\alpha \in \mathbf{P}^{\pi}(\mathbf{p} ; \mathbf{r})} \alpha, \quad \bar{P}^{\pi}(\mathbf{p} ; \mathbf{r})=\sum_{\alpha \in \overline{\mathbf{P}}^{\pi}(\mathbf{p} ; \mathbf{r})}(-1)^{\text {inv } \alpha} \alpha,
\end{aligned}
$$

The following theorem seems technical, but it is actually a combinatorial statement with a wide range of applications to the right-quantum algebra, as we shall see in the following sections. 
Theorem 2.3 Take a matrix $A=\left(a_{i j}\right)_{m \times m}$, non-negative integer vectors $\mathbf{p}, \mathbf{r}$ with $\sum p_{i}=$ $\sum r_{i}$, and permutations $\pi, \sigma \in S_{m}$.

1. Assume that $A$ is right-quantum, i.e. that it has the properties

$$
\begin{aligned}
a_{j k} a_{i k} & =a_{i k} a_{j k}, \\
a_{i k} a_{j l}-a_{j k} a_{i l} & =a_{j l} a_{i k}-a_{i l} a_{j k} \text { for all } k \neq l .
\end{aligned}
$$

Then

$$
O^{\pi}(\mathbf{p} ; \mathbf{r})=P^{\sigma}(\mathbf{p} ; \mathbf{r})
$$

2. Assume that $A$ satisfies (2.2) above, and that $p_{i} \leq 1$ for $i=1, \ldots, m$. Then

$$
\bar{O}^{\pi}(\mathbf{p} ; \mathbf{r})=\bar{P}^{\sigma}(\mathbf{p} ; \mathbf{r}) \text {. }
$$

\section{Proof of Theorem 2.3}

We can replace $\pi$ by id, since this is just relabeling of the variables $a_{i j}$ according to $\pi$. First we construct a natural bijection

$$
\varphi: \mathbf{O}(\mathbf{p} ; \mathbf{r}) \longrightarrow \mathbf{P}^{\sigma}(\mathbf{p} ; \mathbf{r}) .
$$

Take an o-sequence $\alpha=a_{i_{1} j_{1}} a_{i_{2} j_{2}} \cdots a_{i_{n} j_{n}}$, and interpret it as a concatenation of steps. Among the steps $i_{k} \rightarrow j_{k}$ with the lowest $\sigma^{-1}\left(i_{k}\right)$, take the leftmost one. Continue switching this step with the one on the left until it is at the beginning of the sequence. Then take the leftmost step to its right that begins with $j_{k}$, move it to the left until it is the second step of the sequence, and continue this procedure while possible. Now we have a concatenation of a lattice path and a (shorter) o-sequence. Clearly, continuing this procedure on the remaining o-sequence, we are left with a p-sequence with respect to $\sigma$.

EXAMPLE 3.1 The following shows the transformation of

$$
a_{14} a_{12} a_{13} a_{13} a_{14} a_{22} a_{21} a_{23} a_{31} a_{34} a_{33} a_{34} a_{34} a_{34} a_{42} a_{41} a_{42} a_{43} a_{41} a_{41} a_{44}
$$

into

$$
a_{22} a_{21} a_{14} a_{42} a_{23} a_{31} a_{12} a_{34} a_{41} a_{13} a_{33} a_{34} a_{42} a_{34} a_{43} a_{34} a_{41} a_{13} a_{41} a_{14} a_{44}
$$

with respect to $\sigma=2341$. In the first five drawings, the step that must be moved to the left is drawn in bold. In the next three drawings, all the steps that will form a path in the p-sequence are drawn in bold.

Lemma 3.2 The map $\varphi: \mathbf{O}(\mathbf{p} ; \mathbf{r}) \rightarrow \mathbf{P}^{\sigma}(\mathbf{p} ; \mathbf{r})$ constructed above is a bijection.

Proof. Since the above procedure never switches two steps that begin at the same height, there is exactly one o-sequence that maps into a given p-sequence: take all steps starting at height 1 in the p-sequence in the order they appear, then all the steps starting at height 2 in the p-sequence in the order they appear, etc. Clearly, this map preserves the type of the sequence. 


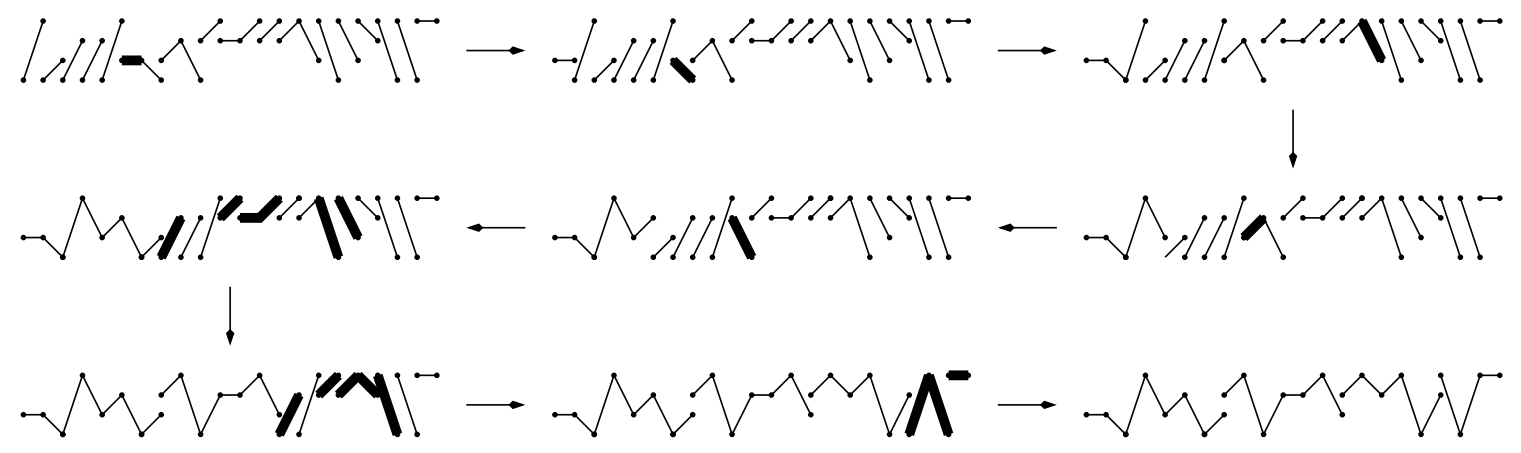

Figure 4: The transformation $\varphi$.

Define a $q$-sequence to be a sequence we get in the transformation of o-sequences into p-sequences with the above procedure (including the o-sequence and the p-sequence). A sequence $a_{i_{1} j_{1}} a_{i_{2} j_{2}} \cdots a_{i_{n} j_{n}}$ is a q-sequence if it is a concatenation of

- some lattice paths with starting heights $i_{k_{s}}$ and ending heights $j_{l_{s}}$ so that $\sigma^{-1}\left(i_{k_{s}}\right) \leq$ $\sigma^{-1}\left(i_{t}\right)$ for all $t \geq k_{s}$, and $i_{t} \neq j_{l_{s}}$ for $t>l_{s}$;

- a lattice path with starting height $i_{k}$ and ending height $j_{k}$ so that $\sigma^{-1}\left(i_{k_{s}}\right) \leq \sigma^{-1}\left(i_{t}\right)$ for all $t \geq k_{s}$; and

- a sequence that is an o-sequence except that the leftmost step with starting height $j_{k}$ can be before some of the steps with starting height $i, \sigma^{-1}(i) \leq \sigma^{-1}\left(j_{k}\right)$.

For a q-sequence $\alpha$, denote by $\psi(\alpha)$ the q-sequence we get by performing the switch described above; for a p-sequence $\alpha$ (where no more switches are needed), $\psi(\alpha)=\alpha$. By construction, the map $\psi$ always switches steps that start on different heights.

For a sequence $a_{i_{1} j_{1}} a_{i_{2} j_{2}} \cdots a_{i_{n} j_{n}}$, define the rank as $\operatorname{inv}\left(i_{1} i_{2} \ldots i_{n}\right)$ (more generally, the rank with respect to $\pi$ is $\left.\operatorname{inv}\left(\pi^{-1}\left(i_{1}\right) \pi^{-1}\left(i_{2}\right) \ldots \pi^{-1}\left(i_{n}\right)\right)\right)$. Clearly, o-sequences are exactly the sequences of rank 0 . Note also that the map $\psi$ increases by 1 the rank of sequences that are not p-sequences.

Write $\mathbf{Q}_{n}^{\sigma}(\mathbf{p} ; \mathbf{r})$ for the union of two sets of sequences of type $(\mathbf{p}, \mathbf{r})$ : the set of all qsequences with rank $n$ and the set of p-sequences (with respect to $\sigma$ ) with rank $<n$; in particular, $\mathbf{O}(\mathbf{p} ; \mathbf{r})=\mathbf{Q}_{0}^{\sigma}(\mathbf{p} ; \mathbf{r})$ and $\mathbf{P}^{\sigma}(\mathbf{p} ; \mathbf{r})=\mathbf{Q}_{N}^{\sigma}(\mathbf{p} ; \mathbf{r})$ for $N$ large enough.

Lemma 3.3 The map $\psi: \mathbf{Q}_{n}^{\sigma}(\mathbf{p} ; \mathbf{r}) \rightarrow \mathbf{Q}_{n+1}^{\sigma}(\mathbf{p} ; \mathbf{r})$ is a bijection for all $n$.

Proof. A q-sequence of rank $n$ which is not a p-sequence is mapped into a q-sequence of rank $n+1$, and $\psi$ is the identity map on p-sequences. This proves that $\psi$ is indeed a map from $\mathbf{Q}_{n}^{\sigma}(\mathbf{p} ; \mathbf{r})$ to $\mathbf{Q}_{n+1}^{\sigma}(\mathbf{p} ; \mathbf{r})$. It is easy to see (and it also follows from the fact that $\varphi=\psi^{N}$ for $N$ large enough) that $\psi$ is injective and surjective.

Proof of Theorem 2.3. Recall that we are assuming that $A$ is right-quantum. Take a qsequence $\alpha$. If $\alpha$ is a p-sequence, then $\psi(\alpha)=\alpha$. Otherwise, assume that $(x-1, i) \rightarrow(x, k)$ 
and $(x, j) \rightarrow(x+1, l)$ are the steps to be switched in order to get $\psi(\alpha)$. If $k=l$, then $\psi(\alpha)=\alpha$ by (2.1). Otherwise, denote by $\beta$ the sequence we get by replacing these two steps with $(x-1, i) \rightarrow(x, l)$ and $(x, j) \rightarrow(x+1, k)$. The crucial observation is that $\beta$ is also a q-sequence, and that its rank is equal to the rank of $\alpha$. Furthermore, $\alpha+\beta=\psi(\alpha)+\psi(\beta)$ because of (2.2). This implies that $\sum \psi(\alpha)=\sum \alpha$ with the sum over all sequences in $\mathbf{Q}_{n}^{\sigma}(\mathbf{p} ; \mathbf{r})$. Repeated application of this shows that

$$
\sum \varphi(\alpha)=\sum \alpha
$$

with the sum over all $\alpha \in \mathbf{O}(\mathbf{p} ; \mathbf{r})$. Because $\varphi$ is a bijection, this finishes the proof of (2.3).

The proof of (2.4) is almost exactly the same. The maps $\bar{\psi}$ and $\bar{\varphi}$ must now move steps to the right instead of to the left. Assume that $(x-1, j) \rightarrow(x, l)$ and $(x, i) \rightarrow(x+1, k)$ are the steps in $\alpha$ we want to switch. The condition $p_{i} \leq 1$ guarantees that $i \neq j$. Denote by $\beta$ the sequence we get by replacing these two steps with $(x-1, i) \rightarrow(x, l)$ and $(x, j) \rightarrow(x+1, k) ; \beta$ is also a q-sequence of the same rank, and because $i \neq j$, its number of inversions differs from $\alpha$ by \pm 1 . The relation (2.2) implies $\alpha-\beta=\bar{\psi}(\alpha)-\bar{\psi}(\beta)$, and this means that $\sum(-1)^{\operatorname{inv} \bar{\psi}(\alpha)} \bar{\psi}(\alpha)=\sum(-1)^{\operatorname{inv} \alpha} \alpha$ and hence also

$$
\sum(-1)^{\operatorname{inv} \bar{\varphi}(\alpha)} \bar{\varphi}(\alpha)=\sum(-1)^{\operatorname{inv} \alpha} \alpha
$$

with the sum over all $\alpha \in \overline{\mathbf{O}}(\mathbf{p} ; \mathbf{r})$.

REMARK 3.4 The transformation $\bar{\varphi}$ for $\pi=\sigma=(m, m-1, \ldots, 1)$ and $\mathbf{p}=\mathbf{r}$ was defined by Foata. See [Lot97, §10.5] for a beautiful presentation of this "first fundamental transformation for arbitrary words".

\section{Matrix inverse formula}

Define the determinant of a matrix $B=\left(b_{i j}\right)_{m \times m}$ as

$$
\operatorname{det} B=\sum_{\pi \in S_{m}}(-1)^{\mathrm{inv} \pi} b_{\pi(1) 1} b_{\pi(2) 2} \cdots b_{\pi(m) m} .
$$

Note that

$$
\operatorname{det} A=\bar{O}^{w_{0}}(\mathbf{1})
$$

where $A=\left(a_{i j}\right)_{m \times m}, w_{0}=m \ldots 21$ and $\mathbf{1}=(1,1, \ldots, 1)$. As the first application of Theorem 2.3, we have $\operatorname{det} A=\bar{P}(\mathbf{1})$ if $A$ is right-quantum; for example, for $m=4$, a graphical representation of $\operatorname{det} A$ for $A$ right-quantum is shown in Figure 5.

Note that

$$
\operatorname{det}(I-A)=\sum_{J}(-1)^{|J|} \operatorname{det} A_{J},
$$

where $J$ runs over all subsets of $[m]$ and $A_{J}$ is the matrix $\left(a_{i j}\right)_{i, j \in J}$. In other words, $\operatorname{det}(I-A)$ is the weighted sum of $a_{\pi\left(i_{1}\right) i_{1}} \cdots a_{\pi\left(i_{k}\right) i_{k}}$ over all permutations $\pi$ of all subsets $\left\{i_{1}, \ldots, i_{k}\right\}$ of $[m]$, with $a_{\pi\left(i_{1}\right) i_{1}} \cdots a_{\pi\left(i_{k}\right) i_{k}}$ weighted by $(-1)^{\operatorname{cyc} \pi}$. 


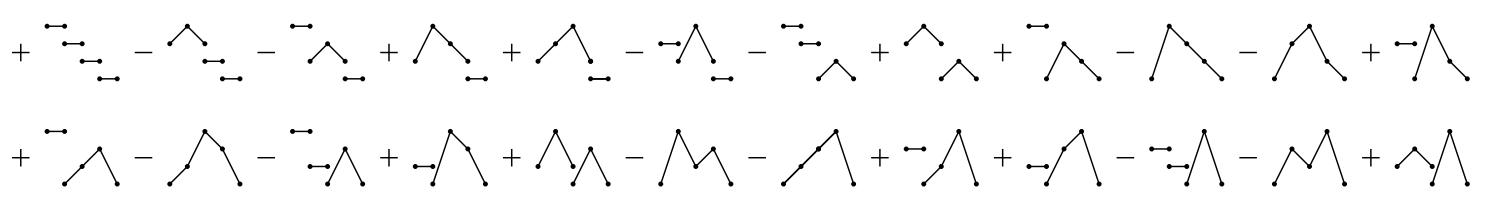

Figure 5: The determinant $\operatorname{det}\left(a_{i j}\right)_{4 \times 4}$.

Theorem 4.1 (right-quantum matrix inverse formula) If $A=\left(a_{i j}\right)_{m \times m}$ is a rightquantum matrix, we have

$$
\left(\frac{1}{I-A}\right)_{i j}=(-1)^{i+j} \cdot \frac{1}{\operatorname{det}(I-A)} \cdot \operatorname{det}(I-A)^{j i}
$$

for all $i, j$.

Here $D^{j i}$ means the matrix $D$ without the $j$-th row and $i$-th column.

We prove the equivalent formula

$$
\operatorname{det}(I-A) \cdot\left(\frac{1}{I-A}\right)_{i j}=(-1)^{i+j} \operatorname{det}(I-A)^{j i}
$$

If $i=j$, the right-hand side is simply (4.1), with $[m]$ replaced by $[m] \backslash\{i\}$, and we can use (2.4) to transform all sequences into bp-sequences with respect to id. Figure 6 shows the right-hand side of (4.2) for $m=4, i=j=3$. If $i \neq j$, the right-hand side of (4.2) is, again by Theorem 2.3, equal to the sum of all bp-sequences with distinct starting and ending heights, with the last lattice path being a path from $i$ to $j$, and with the weight of such a path being 1 if the number of lattice paths is odd, and -1 otherwise. Figure 7 shows this for $m=4, i=2, j=3$.

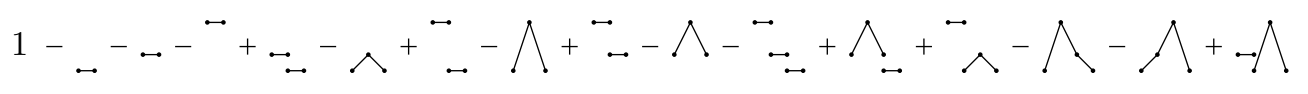

Figure 6: A representation of $\operatorname{det}(I-A)^{33}$.

$$
r-r+V-\vec{r}+\Lambda+\vec{r}-\Lambda-\vec{V}-\Lambda+\Lambda+\Lambda
$$

Figure 7: A representation of $-\operatorname{det}(I-A)^{32}$.

Proof of Theorem 4.1. The left-hand side of (4.2) is equal to

$$
\sum(-1)^{\operatorname{cyc} \alpha} \alpha \cdot \beta,
$$

where the sum runs over all pairs $(\alpha, \beta)$ with the following properties: 
- $\alpha=a_{\pi\left(i_{1}\right) i_{1}} \cdots a_{\pi\left(i_{k}\right) i_{k}}$ for some $i_{1}<\ldots<i_{k}$, and $\pi$ is a permutation of $\left\{i_{1}, \ldots, i_{k}\right\}$; cyc $\alpha$ denotes the number of cycles of $\pi$;

- $\beta$ is a lattice path from $i$ to $j$.

Our goal is to cancel most of the terms and get the right-hand side of (4.2).

Let us divide the pairs $(\alpha, \beta)$ in two groups.

- $(\alpha, \beta) \in \mathcal{G}_{1}$ if no starting or ending height is repeated in $\alpha \cdot \beta$, or the first height that is repeated in $\alpha \cdot \beta$ is a starting height;

- $(\alpha, \beta) \in \mathcal{G}_{2}$ if the first height to be repeated in $\alpha \cdot \beta$ is an ending height.

The sum (4.3) splits into two sums $S_{1}$ and $S_{2}$. Let us discuss each of these in turn.

1. Note that if the first height that is repeated in $\alpha \cdot \beta$ is a starting height, this starting height must be $i$, either as the starting height of the first step of $\beta$ if $\alpha$ contains $i$, or the second occurrence of $i$ as a starting height of $\beta$ if $\alpha$ does not contain $i$. For each $\beta$, we can apply (2.4) with respect to $\sigma=(i, 1, \ldots, i-1, i+1, \ldots, m)$ to the sum

$$
\sum(-1)^{\operatorname{cyc} \alpha} \alpha
$$

over all $\alpha$ with $(\alpha, \beta) \in \mathcal{G}_{1}$. The terms $(-1)^{\text {cyc } \alpha} \alpha \cdot \beta$ that do not include $i$ as a starting height sum up to the right-hand side of (4.2). The terms that do include $i$ either have it in $\alpha$ (and possibly in $\beta$ ) or they have it only in $\beta$. There is an obvious sign-reversing involution between the former and the latter - just move the cycle of $\alpha$ containing $i$ over to $\beta$. This means that $S_{1}$ is equal to the right-hand side of (4.2).

2. Note that the first height $k$ that is repeated in $\alpha \cdot \beta$ as an ending height cannot be $i$. Fix $k$ and a path $\gamma$ from $k$ to $j$. For each path $\gamma^{\prime}$ from $i$ to $k$ without repeated heights, use (2.4) with respect to $\sigma=(k, 1, \ldots, k-1, k+1, \ldots, m)$ on the sum

$$
\sum(-1)^{\operatorname{cyc} \alpha} \alpha
$$

over all $\alpha$ such that $\left(\alpha, \gamma^{\prime} \gamma\right) \in \mathcal{G}_{2}$ and the only repeated height in $\alpha \cdot \gamma^{\prime}$ is the ending height $k$. The sum of

$$
\sum(-1)^{\operatorname{cyc} \alpha} \alpha \cdot \beta
$$

over $(\alpha, \beta) \in \mathcal{G}_{2}, \beta=\gamma^{\prime} \gamma$, and $k$ the only repeated (ending) height in $\alpha \cdot \gamma^{\prime}$, is therefore equal to

$$
\left(\sum \bar{P}^{\sigma}(\mathbf{p} ; \mathbf{r})\right) \cdot \gamma
$$

with

- $\mathbf{p}$ a vector of 1's and 0's, with 1 in the $i$-th entry and the $k$-th entry, and

- $\mathbf{r}$ equal to $\mathbf{p}$ except that the $i$-th entry is 0 and the $k$-th entry is 2 . 
Equation (2.4) of Theorem 2.3 yields

$$
\bar{P}^{\sigma}(\mathbf{p} ; \mathbf{r})=\bar{O}(\mathbf{p} ; \mathbf{r}),
$$

and this is clearly equal to 0 since $\cdots a_{i^{\prime} k} a_{i^{\prime \prime} k} \cdots$ and $\cdots a_{i^{\prime \prime} k} a_{i^{\prime} k} \cdots$ have opposite signs in $\bar{O}(\mathbf{p} ; \mathbf{r})$, and since we have (2.1).

This completes the proof.

REMARK 4.2 The proof consists of two parts. We have to rearrange the steps in each term of the left-hand side of (4.2), and then we use a sign-reversing involution to cancel all the terms except those that appear on the right-hand side.

The first part is trivial if instead of assuming that the variables are right-quantum, we assume that they are commutative or (since we never switch steps that begin at the same height) if they are Cartier-Foata, i.e. if $a_{i k} a_{j l}=a_{j l} a_{i k}$ for $i \neq j$.

The involution itself is also not complicated, and it is worthwhile to restate it more explicitly.

(1) Assume that the first height that is repeated in $\alpha \cdot \beta$ (in notation above) is a starting height $i$. As discussed previously, it can either be the starting height of the first step of $\beta$ if $\alpha$ contains $i$, or the second occurrence of $i$ as a starting height of $\beta$ if $\alpha$ does not contain $i$. The sign-reversing involution between the former and the latter is obvious - just move the cycle of $\alpha$ containing $i$ over to $\beta$ or vice versa.

(2) Assume that the first height that is repeated in $\alpha \cdot \beta$ is an ending height $k$ (which cannot be $i$ ). Write $\beta=\beta^{\prime} \beta^{\prime \prime}$, where $\beta^{\prime}$ is a path from $i$ to $k$ with no repeated heights. The height $k$ can either appear in $\alpha$ (then it appears only as an ending height in $\beta^{\prime}$ ) or not (then it appears once as a starting height and twice as an ending height in $\beta^{\prime}$ ). There exists an obvious involution between the sets of pairs with either of these properties: move the cycle in $\alpha$ starting with $k$ to the end of $\beta^{\prime}$ if $k$ appears as a height in $\alpha$, and move the cycle starting with $k$ from $\beta^{\prime}$ to $\alpha$ otherwise.

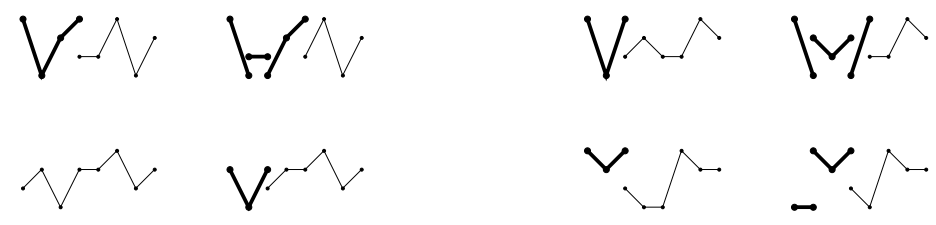

Figure 8: Some pairs that cancel in (4.4).

Figure 8 shows some pairs that are canceled by these involutions for $m=4, i=2$ and $j=3$. The top two pairs belong to $\mathcal{G}_{1}$, and the bottom two pairs belong to $\mathcal{G}_{2}$ (with $k=3$ and $k=1$ respectively). The sequence $\alpha$ in the pair $(\alpha, \beta)$ is drawn in bold. In particular, this proof is much simpler than the one presented in [Foa79]. 


\section{Jacobi ratio theorem}

The proof in the previous section is not only the simplest bijective proof of the matrix inverse formula (but see [Foa79] for an alternative bijective proof in the - less general - Cartier-Foata case, when $a_{i k} a_{j l}=a_{j l} a_{i k}$ for $i \neq j$ ), but also generalizes easily to the proof of Jacobi ratio theorem. This result appears to be new (for either Cartier-Foata or right-quantum matrices), although a variant was proved for general non-commutative variables in [GR91] and for quantum matrices in [KL95].

We need the following proposition.

Proposition 5.1 If the matrix $A=\left(a_{i j}\right)_{m \times m}$ is right-quantum, the matrix $C=\left(c_{i j}\right)_{m \times m}$ with

$$
c_{i j}=\left(\frac{1}{I-A}\right)_{i j}
$$

satisfies (2.2).

Note that $c_{i j}$ is the sum of all paths from $i$ to $j$.

Proof. We need some notation:

- let $O$ denote the sum of $O(\mathbf{p})$ over all $\mathbf{p} \geq 0$, and let $P$ denote the sum of $P(\mathbf{p})$ over all $\mathbf{p} \geq 0$

- the superscript $i$ in front of an expression $E$ means that $E$ contains no variable $a_{i *}$; for example, ${ }^{i} c_{j i}$ denotes the sum of all paths from $j$ to $i$ that reach $i$ exactly once, and ${ }^{i j} O$ is the sum of $O(\mathbf{p})$ over all $\mathbf{p} \geq 0$ with $p_{i}=p_{j}=0$;

- $O_{i}^{j}$ for $i \neq j$ means the sum of $O(\mathbf{p} ; \mathbf{r})$ with $p_{i}=r_{i}+1, p_{j}=r_{j}-1$.

- $O_{i j}^{k}$ for non-equal $i, j, k$ means the sum of $O(\mathbf{p} ; \mathbf{r})$ with $p_{i}=r_{i}+1, p_{j}=r_{j}+1$, $p_{k}=r_{k}-2$.

- $O_{i j}^{k l}$ for non-equal $i, j, k, l$ means the sum of $O(\mathbf{p} ; \mathbf{r})$ with $p_{i}=r_{i}+1, p_{j}=r_{j}+1$, $p_{k}=r_{k}-1, p_{l}=r_{l}-1$.

We have to prove $c_{i k} c_{j l}+c_{i l} c_{j k}=c_{j k} c_{i l}+c_{j l} c_{i k}$ for $k<l$. Let us investigate three possible cases:

- Take $i=k, j=l$. First let us prove that $c_{j j}{ }^{j} c_{i j}=c_{i j}$. To see this, use (2.3) on $O_{i}^{j}$ twice, once with respect to $\pi=(i, j, 1, \ldots, i-1, i+1, \ldots, j-1, j+1, \ldots, m)$ and once with respect to $\sigma=(j, i, 1, \ldots, i-1, i+1, \ldots, j-1, j+1, \ldots, m)$. We get

$$
O_{i}^{j}=c_{i j}{ }^{j} P^{\pi} \quad \text { and } \quad O_{i}^{j}=c_{j j}{ }^{j} c_{i j}{ }^{j} P^{\pi}
$$

and since ${ }^{j} P^{\pi}$ is invertible in $\mathcal{A}$ (its constant term is 1 ), we have

$$
c_{i j}=c_{j j}{ }^{j} c_{i j}
$$


By using (2.3) on $O$ twice, once with respect to $\pi$ and once with respect to $\sigma$, we get

$$
O=c_{i i}{ }^{i} c_{j j}{ }^{i j} P=c_{j j}{ }^{j} c_{i i}{ }^{i j} P .
$$

Furthermore, (5.2) yields

$$
c_{i i}{ }^{i} c_{j j}=c_{i i}\left(c_{j j}-{ }^{i} c_{j i} c_{i j}\right)=c_{i i} c_{j j}-\left(c_{i i}{ }^{i} c_{j i}\right) c_{i j}=c_{i i} c_{j j}-c_{j i} c_{i j}
$$

and similarly

$$
c_{j j}{ }^{j} c_{i i}=c_{j j} c_{i i}-c_{i j} c_{j i}
$$

so (5.3) gives

$$
c_{i i} c_{j j}-c_{j i} c_{i j}=c_{j j} c_{i i}-c_{i j} c_{j i} .
$$

- Take $i \neq k, j=l$ (and the case $i=k, j \neq l$ is proved analogously). First note that

$$
O_{i j}^{k}=c_{i k}{ }^{k} c_{j k}{ }^{k} P=c_{j k}{ }^{k} c_{i k}{ }^{k} P
$$

yields

$$
c_{i k}{ }^{k} c_{j k}=c_{j k}{ }^{k} c_{i k}
$$

Use (2.3) on $O_{j}^{k}$ twice, once with respect to $\pi$ and once with respect to $\tau=$ $(j, 1, \ldots, j-1, j+1, \ldots, m)$. We get

$$
O_{j}^{k}=c_{i i}{ }^{i} c_{j k}{ }^{i k} P+c_{i k}{ }^{k} c_{j i}{ }^{i k} P \quad \text { and } \quad O_{j}^{k}=c_{j k}{ }^{k} P=c_{j k}{ }^{k} c_{i i}{ }^{i k} P .
$$

But then

$$
c_{i i}{ }^{i} c_{j k}+c_{i k}{ }^{k} c_{j i}=c_{j k}{ }^{k} c_{i i}
$$

implies

$$
c_{i i}\left(c_{j k}-{ }^{i} c_{j i} c_{i k}\right)+c_{i k}\left(c_{j i}-{ }^{k} c_{j k} c_{k i}\right)=c_{j k}\left(c_{i i}-{ }^{k} c_{i k} c_{k i}\right)
$$

and

$$
c_{i i} c_{j k}+c_{i k} c_{j i}=c_{j k} c_{i i}+c_{i i}{ }^{i} c_{j i} c_{i k}+\left(c_{i k}{ }^{k} c_{j k}-c_{j k}{ }^{k} c_{i k}\right) c_{k i},
$$

and so (5.2) and (5.4) imply

$$
c_{i i} c_{j k}+c_{i k} c_{j i}=c_{j k} c_{i i}+c_{j i} c_{i k}
$$

- Assume that $i \neq k, j \neq l$. Then

$$
\begin{gathered}
O_{i j}^{k l}=c_{i k}{ }^{k} c_{j l}{ }^{k l} P+c_{i l}{ }^{l} c_{j k}{ }^{k l} P=c_{j k}{ }^{k} c_{i l}{ }^{k l} P+c_{j l}{ }^{l} c_{i k}{ }^{k l} P, \\
c_{i k}\left(c_{j l}-{ }^{k} c_{j k} c_{k l}\right)+c_{i l}\left(c_{j k}-{ }^{l} c_{j l} c_{l k}\right)=c_{j k}\left(c_{i l}-{ }^{k} c_{i k} c_{k l}\right)+c_{j l}\left(c_{i k}-{ }^{l} c_{i l} c_{l k}\right),
\end{gathered}
$$

and (5.4) imply

$$
c_{i k} c_{j l}+c_{i l} c_{j k}=c_{j k} c_{i l}+c_{j l} c_{i k} .
$$

This completes the proof. 
REMARK 5.2 It is not hard to see that $C$ is actually a right-quantum matrix. We do not need this fact, however.

Theorem 5.3 (right-quantum Jacobi ratio theorem) Take $I, J \subseteq[m]$ with $|I|=|J|$. If $A=\left(a_{i j}\right)_{m \times m}$ is right-quantum and $C=\left(c_{i j}\right)_{m \times m}$ is given by

$$
c_{i j}=\left(\frac{1}{I-A}\right)_{i j} \text {, }
$$

then

$$
\operatorname{det} C_{I, J}=(-1)^{\sum_{i \in I} i+\sum_{j \in J} j} \cdot \frac{1}{\operatorname{det}(I-A)} \cdot \operatorname{det}(I-A)^{J, I} .
$$

In particular,

$$
\operatorname{det}\left(\frac{1}{I-A}\right)=\frac{1}{\operatorname{det}(I-A)} \text {. }
$$

Proof. We only sketch the proof as it is very similar to the proof of Theorem 4.1 once we have Proposition 5.1, and we assume that $I=J$ as this makes the reasoning slightly simpler. Use (2.4) (we can do that because of Proposition 5.1) on $\operatorname{det} C_{I}$; for a permutation $\pi \in S_{m}$ with cyclic structure $\left(i_{1}^{1} i_{2}^{1} \ldots i_{k_{1}}^{1}\right)\left(i_{1}^{2} i_{2}^{2} \ldots i_{k_{2}}^{2}\right) \cdots\left(i_{1}^{l} i_{2}^{l} \ldots i_{k_{l}}^{l}\right)$ (where the first element of each cycle is the smallest, and where starting elements of cycles are increasing), we get the term

$$
(-1)^{\operatorname{inv} \pi}\left(c_{i_{1}^{l}, i_{2}^{l}} \cdots c_{i_{k}, i_{1}^{l}}\right) \cdots\left(c_{i_{1}^{2}, i_{2}^{2}} \cdots c_{i_{k_{2}}^{2}, i_{1}^{2}}\right)\left(c_{i_{1}^{1}, i_{2}^{1}} \cdots c_{i_{k_{1}}^{1}, i_{1}^{1}}\right)
$$

For each selection of paths (in variables $a_{i j}$ )

$$
i_{1}^{l} \rightarrow i_{2}^{l}, \ldots, i_{k_{l}}^{l} \rightarrow i_{1}^{l}, \ldots, i_{1}^{2} \rightarrow i_{2}^{2}, \ldots, i_{k_{2}}^{2} \rightarrow i_{1}^{2}, i_{1}^{1} \rightarrow i_{2}^{1}, \ldots, i_{k_{1}}^{1} \rightarrow i_{1}^{1},
$$

this yields a concatenation of (possibly empty) lattice paths from $i_{1}^{l}$ to $i_{1}^{l}$, $i_{1}^{l-1}$ to $i_{1}^{l-1}$, etc., with exactly one starting height $i_{s}^{t}$ for every $s$ marked on each path $i_{1}^{t} \rightarrow i_{1}^{t}$. For example, take $m=5, I=\{1,2,4\}$, and $\pi=\left(\begin{array}{l}124 \\ 421\end{array}\right)$. The term of $\operatorname{det} C_{I}$ corresponding to $\pi$ is $-c_{22} c_{14} c_{41}$, and some of the sequences (without the minus sign) corresponding to this term are depicted in Figure 9. Note the empty path corresponding to $c_{22}$ in the second example. When we multiply $\operatorname{det} C_{I}$ on the left by $\operatorname{det}(I-A)$, we get a sum

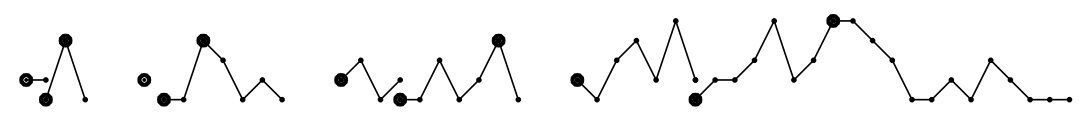

Figure 9: Some sequences in $c_{22} c_{14} c_{41}$.

$$
\sum(-1)^{\operatorname{cyc} \alpha+\operatorname{inv} \beta} \alpha \cdot \beta,
$$

where the sum runs over all pairs $(\alpha, \beta)$ with the following properties: 
- $\alpha=a_{\pi\left(i_{1}\right) i_{1}} \cdots a_{\pi\left(i_{k}\right) i_{k}}$ for some $i_{1}<\ldots<i_{k}$, and $\pi$ is a permutation of $\left\{i_{1}, \ldots, i_{k}\right\}$; cyc $\alpha$ denotes the number of cycles of $\pi$;

- $\beta$ is a concatenation of lattice path from $i_{1}^{l}$ to $i_{l}^{1}, i_{1}^{l-1}$ to $i_{1}^{l-1}$ with exactly one starting height $i_{s}^{t}$ marked on each path $i_{1}^{t} \rightarrow i_{1}^{t}$, where

$$
\sigma=\left(i_{1}^{1} i_{2}^{1} \ldots i_{k_{1}}^{1}\right)\left(i_{1}^{2} i_{2}^{2} \ldots i_{k_{2}}^{2}\right) \cdots\left(i_{1}^{l} i_{2}^{l} \ldots i_{k_{l}}^{l}\right)
$$

and inv $\beta$ denotes the number of inversions of $\sigma$.

The cancellation process described in the proof of the matrix inverse formula applies here almost verbatim, and this shows that $\operatorname{det}(I-A) \cdot \operatorname{det} C_{I}$ is equal to $\operatorname{det}(I-A)^{I, I}$.

\section{A generalization of the MacMahon master theorem}

MacMahon master theorem is a result classically used for proofs of binomial identities. In this section, we see that the bijection used in the proof of Theorem 2.3 gives a far-reaching extension.

Theorem 6.1 Choose a right-quantum matrix $A=\left(a_{i j}\right)_{m \times m}$, and let $x_{1}, \ldots, x_{m}$ be commuting variables that commute with $a_{i j}$. For $\mathbf{p}, \mathbf{r} \geq \mathbf{0}$, denote the coefficient

$$
\left[\mathbf{x}^{\mathbf{r}}\right] \prod_{i=1}^{m}\left(a_{i 1} x_{1}+\ldots+a_{i m} x_{m}\right)^{p_{i}}
$$

by $G(\mathbf{p} ; \mathbf{r})$, and choose an integer vector $\mathbf{d}$ with $\sum d_{i}=0$. Then the generating function

$$
F_{A, \mathbf{d}}(\mathbf{t})=\sum_{\mathbf{p}=\mathbf{r}+\mathbf{d}} G(\mathbf{p} ; \mathbf{r}) \mathbf{t}^{\mathbf{p}}
$$

is a (non-commutative) rational function (with coefficients polynomials in $a_{i j}$ ) in $t_{1}, \ldots, t_{m}$ that can be evaluated as follows. Write $T=\operatorname{diag} \mathbf{t}$. Denote by $\mathcal{M}$ the multiset of all $i$ with $d_{i}<0$, with each $i$ appearing $-d_{i}$ times, and by $S(\mathcal{M})$ the set of all permutations of the multiset $\mathcal{M}$; denote by $\mathcal{N}=\left(N_{1}, N_{2}, \ldots, N_{d}\right)$ (in non-decreasing order) the multiset of all $i$ with $d_{i}>0$, with each $i$ appearing $d_{i}$ times. Note that $\mathcal{M}$ and $\mathcal{N}$ have the same cardinality $\delta$; write $M$ (respectively $N$ ) for the sum of all elements of $\mathcal{M}$ (respectively $\mathcal{N}$ ) counted with their multiplicities. For $\pi=\pi_{1} \cdots \pi_{\delta} \in S(\mathcal{M})$, let $I_{\pi}^{k}($ for $1 \leq k \leq \delta)$ be the set $\left\{\pi_{1}, \ldots, \pi_{k}\right\}$, write $\varepsilon_{\pi}^{k}$ for the size of the intersection of $I_{\pi}^{k-1}$ and the open interval between $\pi_{k}$ and $N_{k}$, and write $J_{\pi}^{k}=\left(I_{\pi}^{k} \backslash\left\{\pi_{k}\right\}\right) \cup\left\{N_{k}\right\}$. Then

$$
F_{A, \mathbf{d}}(\mathbf{t})=\frac{(-1)^{M+N}}{\operatorname{det}(I-T A)} \sum_{\pi \in S(\mathcal{M})} \prod_{k=1}^{\delta}(-1)^{\varepsilon_{\pi}^{k}} \cdot \operatorname{det}(I-T A)^{I_{\pi}^{k}, J_{\pi}^{k}} \cdot \frac{1}{\operatorname{det}(I-T A)^{I_{\pi}^{k}, I_{\pi}^{k}}} .
$$

Before proving Theorem 6.1, let us show some examples. 
ExAmple 6.2 If $\mathbf{d}=\mathbf{0}, \mathcal{M}=\mathcal{N}=\varnothing, S(\mathcal{M})=\{\varnothing\}, M=N=0, \delta=0$ and

$$
F_{A, \mathbf{0}}(\mathbf{t})=\frac{1}{\operatorname{det}(I-T A)}
$$

which is the right-quantum MacMahon master theorem.

EXAmPle 6.3 Take

$$
A=\left(\begin{array}{llll}
2 & 1 & 4 & 2 \\
3 & 2 & 4 & 3 \\
3 & 4 & 1 & 1 \\
1 & 3 & 5 & 5
\end{array}\right)
$$

and $\mathbf{d}=(1,-2,2,-1)$. i.e.

$$
F=F_{A, \mathbf{d}}(\mathbf{t})=40 t v^{2}+262 t^{2} v^{2}+128 t u v^{2}+312 t v^{3}+251 t v^{2} w+\ldots
$$

where we write $t, u, v, w$ for $t_{1}, t_{2}, t_{3}, t_{4}$. We have $\mathcal{M}=(2,2,4), S(\mathcal{M})=\{224,242,422\}$, $\mathcal{N}=(1,3,3), \delta=3, M=8, N=7, I_{224}^{1}=\{2\}, I_{224}^{2}=\{2\}, I_{224}^{3}=\{2,4\}, I_{242}^{1}=\{2\}$, $I_{242}^{2}=\{2,4\}, I_{242}^{3}=\{2,4\}, I_{422}^{1}=\{4\}, I_{422}^{2}=\{2,4\}, I_{422}^{3}=\{2,4\}, \varepsilon_{\pi}^{i}=0$ for all $\pi$ and $i$, $J_{224}^{1}=\{1\}, J_{224}^{2}=\{3\}, J_{224}^{3}=\{3,4\}, J_{242}^{1}=\{1\}, J_{242}^{2}=\{2,3\}, J_{242}^{3}=\{3,4\}, J_{422}^{1}=\{1\}$, $J_{422}^{2}=\{3,4\}, J_{422}^{3}=\{3,4\}$. Therefore

$$
\begin{aligned}
F=-\frac{1}{\operatorname{det}(I-T A)}\left(\frac{\operatorname{det}(I-T A)^{2,1}}{\operatorname{det}(I-T A)^{2,2}} \frac{\operatorname{det}(I-T A)^{2,3}}{\operatorname{det}(I-T A)^{2,2}} \frac{\operatorname{det}(I-T A)^{24,34}}{\operatorname{det}(I-T A)^{24,24}}+\right. \\
\frac{\operatorname{det}(I-T A)^{2,1}}{\operatorname{det}(I-T A)^{2,2}} \frac{\operatorname{det}(I-T A)^{24,23}}{\operatorname{det}(I-T A)^{24,24}} \frac{\operatorname{det}(I-T A)^{24,34}}{\operatorname{det}(I-T A)^{24,24}}+ \\
\left.\frac{\operatorname{det}(I-T A)^{4,1}}{\operatorname{det}(I-T A)^{4,4}} \frac{\operatorname{det}(I-T A)^{24,34}}{\operatorname{det}(I-T A)^{24,24}} \frac{\operatorname{det}(I-T A)^{24,34}}{\operatorname{det}(I-T A)^{24,24}}\right)= \\
=-\frac{D_{24,34}\left(D_{2,1} D_{2,3} D_{4,4} D_{24,24}+D_{2,1} D_{2,2} D_{4,4} D_{24,23} D_{24,34}+D_{4,1} D_{2,2}^{2} D_{24,34}\right)}{D D_{2,2}^{2} D_{4,4} D_{24,24}^{2}},
\end{aligned}
$$

where

$$
\begin{aligned}
D=\operatorname{det}(I-T A)= & 1-2 t-2 u-v-5 w+t u-10 t v+8 t w-14 u v+ \\
& u w-5 t u v-4 t u w+28 t v w+17 u v w+46 t u v w, \\
D_{2,1}=\operatorname{det}(I-T A)^{2,1}= & -t-15 t v-t w+34 t v w \\
D_{2,2}=\operatorname{det}(I-T A)^{2,2}= & 1-2 t-v-5 w-10 t v+8 t w+28 t v w, \\
D_{2,3}=\operatorname{det}(I-T A)^{2,3}= & -4 v+5 t v+17 v w-30 t v w \\
D_{4,1}=\operatorname{det}(I-T A)^{4,1}= & -2 t+t u-2 t v-13 t u v \\
D_{4,4}=\operatorname{det}(I-T A)^{4,4}= & 1-2 t-2 u-v+t u-10 t v-14 u v-5 t u v, \\
D_{24,23}=\operatorname{det}(I-T A)^{24,23}= & -v-4 t v \\
D_{24,24}=\operatorname{det}(I-T A)^{24,24}= & 1-2 t-v-10 t v \\
D_{24,34}=\operatorname{det}(I-T A)^{24,34}= & -4 v+5 t v .
\end{aligned}
$$

Since we are dealing with complex variables, we do not have to worry about the order of multiplication. 
Proof of Theorem 6.1. Fix non-negative integer vectors $\mathbf{p}, \mathbf{r}$ with $\mathbf{p}=\mathbf{r}+\mathbf{d}$, and use (2.3) on $\mathbf{O}(\mathbf{p} ; \mathbf{r})$ with respect to the permutation $\sigma=i_{1} \cdots i_{s} j_{1} \cdots, j_{t}$, where $i_{1}<i_{2}<\ldots<i_{s}$ form the underlying set of $\mathcal{N}$ (in other words, $\left\{i_{1}, \ldots, i_{s}\right\}=\left\{i: d_{i}>0\right\}$ ) and $j_{1}<j_{2}<$ $\ldots<j_{t}$ are the remaining elements of $\{1, \ldots, m\}$. A path sequence in $\mathbf{P}^{\sigma}(\mathbf{p} ; \mathbf{r})$ has the following structure. The first path starts at $N_{1}=i_{1}$ and ends at one of the heights in $\mathcal{M}$; the second path starts at $N_{2}$ (which is $i_{1}$ if $d_{i_{1}}>1$, and $i_{2}$ if $d_{i_{1}}=1$ ), and ends at one of the heights in $\mathcal{M}$, and it does not include the ending height of the previous path except possibly as the ending height. In general, the $k$-th path starts at $N_{k}$ and ends at one of the heights in $\mathcal{M}$, and does not contain any of the ending heights of previous paths except possibly as the ending height. All together, the ending heights of these $\delta$ paths form a permutation of $\mathcal{M}$, which explains why $F_{A, \mathbf{d}}(\mathbf{t})$ is written as a sum over $\pi \in S(\mathcal{M})$. After these paths, we have a balanced path sequence that does not include any height in $\mathcal{M}$. Now choose $\pi=\pi_{1} \cdots \pi_{\delta} \in S(\mathcal{M})$, and look at all the p-sequences in $\mathbf{P}^{\sigma}(\mathbf{p} ; \mathbf{r}$ ) (for all $\mathbf{p}, \mathbf{r} \geq \mathbf{0}$ with $\mathbf{p}=\mathbf{r}+\mathbf{d}$ ) whose first $\delta$ ending heights of paths are $\pi_{1}, \ldots, \pi_{\delta}$ (in this order). The $k$-th path is a path from $N_{k}$ to $\pi_{k}$, and it does not include $\pi_{1}, \ldots, \pi_{k-1}$ except possibly as an ending height. By the matrix inverse formula, such paths, weighted by $\mathbf{t}^{\mathbf{p}_{\mathbf{k}}}$ where $\left(\mathbf{p}_{\mathbf{k}}, \mathbf{r}_{\mathbf{k}}\right)$ is the type of the path, are enumerated by

$$
\pm \frac{1}{\operatorname{det}(I-T A)^{I_{\pi}^{k-1}, I_{\pi}^{k-1}}} \cdot \operatorname{det}(I-T A)^{I_{\pi}^{k}, J_{\pi}^{k}}
$$

and a simple consideration shows that the sign is $(-1)^{N_{k}+\pi_{k}+\varepsilon_{\pi}^{k}}$. The balanced path sequences that do not include heights from $\mathcal{M}$ are enumerated by

$$
\begin{gathered}
\left(\frac{1}{\operatorname{det}(I-T A)^{I_{\pi}^{\delta}}} \cdot \operatorname{det}(I-T A)^{I_{\pi}^{\delta} \cup\left\{j_{1}\right\}}\right) \cdot\left(\frac{1}{\operatorname{det}(I-A)^{I_{\pi}^{\delta} \cup\left\{j_{1}\right\}}} \cdot \operatorname{det}(I-A)^{I_{\pi}^{\delta} \cup\left\{j_{1}, j_{2}\right\}}\right) \cdots \frac{1}{1-a_{j_{t} j_{t}}}= \\
=\frac{1}{\operatorname{det}(I-T A)^{I_{\pi}^{\delta}}},
\end{gathered}
$$

where we wrote $\operatorname{det}(I-T A)^{I}$ instead of $\operatorname{det}(I-T A)^{I, I}$. Formula (6.1) follows.

Proof of (1.1). Let us denote the sum we are trying to calculate by $S(n)$. Clearly,

$$
\begin{gathered}
{\left[x^{n+1} y^{n+1} z^{n-2}\right](z-y)^{n}(x-z)^{n}(y-x)^{n}=\left[x y z^{-2}\right]\left(1-\frac{y}{z}\right)^{n}\left(1-\frac{z}{x}\right)^{n}\left(1-\frac{x}{y}\right)^{n}=} \\
=\left[x y z^{-2}\right] \sum_{i, j, k}(-1)^{i+j+k}\left(\begin{array}{c}
n \\
i
\end{array}\right)\left(\begin{array}{c}
n \\
j
\end{array}\right)\left(\begin{array}{c}
n \\
k
\end{array}\right)\left(\frac{x}{y}\right)^{i}\left(\frac{y}{z}\right)^{j}\left(\frac{z}{x}\right)^{k}=S(n),
\end{gathered}
$$

and so we have to use Theorem 6.1 for

$$
A=\left(\begin{array}{ccc}
0 & -1 & 1 \\
1 & 0 & -1 \\
-1 & 1 & 0
\end{array}\right)
$$

and $\mathbf{d}=(-1,-1,2)$. We get

$$
\sum_{\mathbf{p}=\mathbf{r}+\mathbf{d}} G(\mathbf{p} ; \mathbf{r}) t^{p_{1}} u^{p_{2}} v^{p_{3}}=\frac{-v^{2}(1+t)}{(1+t v)(1+t u+t v+u v)}+\frac{-v^{2}(1-u)}{(1+u v)(1+t u+t v+u v)}
$$


and, after using some obvious symmetry,

$$
\begin{gathered}
S(n)=2\left[t^{n} u^{n} v^{n}\right]\left(\frac{-v^{2}}{(1+t v)(1+t u+t v+u v)}\right)= \\
=2 \sum_{i, j, k, l}(-1)^{l+1}(t v)^{l} v^{2}\left(\begin{array}{c}
i+j+k \\
i, j, k
\end{array}\right)(-1)^{i+j+k}(t u)^{i}(t v)^{j}(u v)^{k},
\end{gathered}
$$

with the sum over all $i, j, k, l \geq 0$ with $l+i+j=n, i+j=n, l+2+j+k=n$, i.e. $S(n)=0$ if $n$ is odd and

$$
S(2 m)=\frac{2(-1)^{m}}{(m+1) !(m-1) !} \sum_{l=0}^{m-1} \frac{(3 m-1-l) !}{(m-1-l) !}=2(-1)^{m}\left(\begin{array}{c}
2 m \\
m-1
\end{array}\right) \sum_{l=0}^{m-1}\left(\begin{array}{c}
3 m-1-l \\
m-1-l
\end{array}\right)
$$

therefore $S(2 m)=2(-1)^{m}\left(\begin{array}{c}2 m \\ m-1\end{array}\right)\left(\begin{array}{c}3 m \\ m-1\end{array}\right)$, since every $(m-1)$-subset of $\{1, \ldots, 3 m\}$ consists of elements $\{1, \ldots, l\}$ and an $(m-1-l)$-subset of $\{l+2, \ldots, 3 m\}$ for a uniquely determined $l$.

\section{Final remarks}

Some of the results (Theorems 2.3 and 4.1) have natural $q$ - and q-analogues; these can either be proved by the $1=q$ and $1=q_{i j}$ principles (see [FH, §3] and [KP07, Lemma 12.4]) or by some straightforward bookkeeping, cf. [KP07, §§5-8]. However, Theorems 5.3 and 6.1 do not seem to extend to a formula for general $q$ or $q_{i j}$.

As can be seen from the proof of Theorem 4.1, the formula

$$
\left(\frac{1}{I-A}\right)_{m m}=\frac{1}{\operatorname{det}(I-A)} \cdot \operatorname{det}(I-A)^{m m}
$$

holds even if (2.1) and (2.2) hold only for $k, l \neq m$. This fact (in the more general q-rightquantum case) was used in the proof of q-Cartier-Foata and q-right-quantum Sylvester's determinant identity [Kon07, Theorem 1.3].

It is easy to prove that the variables $c_{i j}$ defined by (5.1) satisfy (2.1), i.e. that the matrix $C=\left(c_{i j}\right)_{m \times m}$ is right-quantum. We do not need this fact, however.

Even though the statement of Theorem 6.1 appears rather intricate even in the commutative case, it is very easy to find the generating function with the help of a computer. A Mathematica package genmacmahon.m that calculates $F_{A, \mathbf{d}}(\mathbf{t})$ for a commutative matrix $A=\left(a_{i j}\right)_{m \times m}$ and an integer vector $\mathbf{d}=\left(d_{1}, \ldots, d_{m}\right)$ with $\sum d_{i}=0$ is available at http://www-math.mit.edu/ ${ }^{\sim}$ konvalinka/genmacmahon.m (read in the package with $<$ genmacmahon.m and write $\mathrm{F}[\mathrm{A}, \mathrm{d}, \mathrm{t}])$, and it would be easy to adapt this to the noncommutative situation. 
It would be nice to use Theorem 6.1 to prove

$$
\sum_{i=k}^{n-k}(-1)^{i}\left(\begin{array}{c}
n \\
i-k
\end{array}\right)\left(\begin{array}{c}
n \\
i
\end{array}\right)\left(\begin{array}{c}
n \\
i+k
\end{array}\right)=\frac{(-1)^{m}(2 m) !^{2}(3 m) !}{m !(m-k) !(m+k) !(2 m-k) !(2 m+k) !},
$$

which can be established by the WZ method [PWZ96]. The author proved that

$$
S_{k}(n)=\sum_{i=k}^{n-k}(-1)^{i}\left(\begin{array}{c}
n \\
i-k
\end{array}\right)\left(\begin{array}{c}
n \\
i
\end{array}\right)\left(\begin{array}{c}
n \\
i+k
\end{array}\right)
$$

satisfies $S_{k}(n)=0$ if $n$ is odd and, if $k \geq 1$,

$$
S_{k}(2 m)=2 \sum_{j=1}^{k}\left(\begin{array}{c}
2 k-j-1 \\
k-1
\end{array}\right) \sum_{i=0}^{\lfloor j / 2\rfloor}(-1)^{m-i}\left(\begin{array}{c}
j \\
2 i
\end{array}\right)\left(\begin{array}{c}
3 m-i+j-k \\
m-i
\end{array}\right)\left(\begin{array}{c}
2 m \\
m+k-i
\end{array}\right)
$$

and it is easy to see for small $k$ that this is equal to the right-hand side of (7.1). Christian Krattenthaler has pointed out that the identity (7.1) is a special case of

$$
{ }_{3} F_{2}(a, b, c ; 1+a-b, 1+a-c ; 1)=\frac{\Gamma\left(1+\frac{a}{2}\right) \Gamma(1+a-b) \Gamma(1+a-c) \Gamma\left(1+\frac{a}{2}-b-c\right)}{\Gamma(1+a) \Gamma\left(1+\frac{a}{2}-b\right) \Gamma\left(1+\frac{a}{2}-c\right) \Gamma(1+a-b-c)},
$$

a more general form of Dixon's identity.

It is easy to find the "dual" version of Theorem 6.1, namely to calculate

$$
G_{A, \mathbf{d}}(\mathbf{t})=\sum_{\mathbf{p}=\mathbf{r}+\mathbf{d}} G(\mathbf{p} ; \mathbf{r}) \mathbf{t}^{\mathbf{r}}:
$$

paths from $N_{k}$ to $\pi_{k}$ that do not include $\pi_{1}, \ldots, \pi_{k-1}$ except possibly as an ending height, weighted by $\mathbf{t}^{\mathbf{r}_{\mathbf{k}}}$, where $\left(\mathbf{p}_{\mathbf{k}}, \mathbf{r}_{\mathbf{k}}\right)$ is the type of the path, are enumerated by

$$
\pm \frac{1}{\operatorname{det}(I-A T)^{I_{\pi}^{k-1}, I_{\pi}^{k-1}}} \cdot \operatorname{det}(I-A T)^{I_{\pi}^{k}, J_{\pi}^{k}}
$$

Therefore, in the notation of Theorem 6.1,

$$
G_{A, \mathbf{d}}(\mathbf{t})=\frac{(-1)^{M+N}}{\operatorname{det}(I-A T)} \sum_{\pi \in S(\mathcal{M})} \prod_{k=1}^{\delta}(-1)^{\varepsilon_{\pi}^{k}} \cdot \operatorname{det}(I-A T)^{I_{\pi}^{k}, J_{\pi}^{k}} \cdot \frac{1}{\operatorname{det}(I-A T)^{I_{\pi}^{k}, I_{\pi}^{k}}} .
$$

Acknowledgment. The author is grateful to Igor Pak for his help and support. 


\section{References}

[FH] D. Foata and G.-N. Han, A basis for the right quantum algebra and the " $1=q "$ principle, arXive:math.CO/0603463, to appear in J. Algebraic Combin.

[FH07a] _ A new proof of the Garoufalidis-Lê-Zeilberger quantum MacMahon Master Theorem, J. Algebra 307 (2007), no. 1, 424-431.

[FH07b] _ Specializations and extensions of the quantum MacMahon Master Theorem, Linear Algebra Appl. 423 (2007), no. 2-3, 445-455.

[Foa65] D. Foata, Étude algébrique de certains problèmes d'analyse combinatoire et du calcul des probabilités, Publ. Inst. Statist. Univ. Paris 14 (1965), 81-241.

[Foa79] A Noncommutative Version of the Matrix Inversion Formula, Adv. Math. 31 (1979), 330-349.

[GLZ06] S. Garoufalidis, T. Tq Lê, and D. Zeilberger, The quantum MacMahon master theorem, Proc. Natl. Acad. Sci. USA 103 (2006), no. 38, 13928-13931 (electronic).

[GR91] I. M. Gelfand and V. S. Retakh, Determinants of matrices over noncommutative rings, Funct. Anal. Appl. 25 (1991), no. 2, 91-102.

[HL07] P. H. Hai and M. Lorenz, Koszul algebras and the quantum MacMahon master theorem, Bull. London Math. Soc. 277 (2007), 667-676.

[KL95] D. Krob and B. Leclerc, Minor Identities for Quasi-Determinants and Quantum Determinants, Commun. Math. Phys. 169 (1995), 1-23.

[Kon07] M. Konvalinka, Non-commutative Sylvester's determinantal identity, Electron. J. Combin. 14 (2007), no. 1, Research Paper 42, 29 pp. (electronic).

[KP07] M. Konvalinka and I. Pak, Non-commutative extensions of MacMahon's Master Theorem, Adv. Math. 216 (2007), 29-61.

[Lot97] M. Lothaire, Combinatorics on words, Cambridge Mathematical Library, Cambridge University Press, Cambridge, 1997.

[Man88] Yu. I. Manin, Quantum groups and noncommutative geomtry, CRM, Université de Montréal, QC, 1988.

[Man89] , Multiparameter quantum deformations of the linear supergroup, Comm. Math. Phys. 123 (1989), 163-175.

[PWZ96] M Petkovšek, H. S. Wilf, and D. Zeilberger, $A=B$, A K Peters, Ltd., Wellesley, MA, 1996. 